Quim. Nova, Vol. 34, No. 6, 962-967, 2011

\title{
APLICAÇÃO DA CROMATOGRAFIA GASOSA BIDIMENSIONAL ABRANGENTE COM MICRODETECTOR DE CAPTURA DE ELÉTRONS PARA DETERMINAÇÃO DE AGROTÓXICOS EM SEDIMENTOS
}

\author{
Juliana Macedo da Silva, Cláudia Alcaraz Zini e Elina Bastos Caramão* \\ Instituto de Química, Universidade Federal do Rio Grande do Sul, Av. Bento Gonçalves, 9500, 91501-970 Porto Alegre - RS, Brasil
}

Recebido em 27/9/10; aceito em 16/1/11; publicado na web em 29/3/11

\begin{abstract}
APPLICATION OF COMPREHENSIVE TWO-DIMENSIONAL GAS CHROMATOGRAPHY WITH MICRO-ELECTRON CAPTURE DETECTION FOR DETERMINATION OF PESTICIDES IN SEDIMENTS. Analysis of seven pesticides in sediments was successfully achieved using comprehensive two-dimensional gas chromatography with micro-electron capture detection, as it provided higher sensitivity and less matrix interference. Repeatability and intermediate precision of peak areas and heights were less than $4 \%$ and the recovery percentage for the analytes ranged from 52 to $115 \%$. Instrumental LOD and LOQ were in the range of 0.60 to $2.31 \mu \mathrm{g} \mathrm{L}^{-1}$ and 1.83 to $5.62 \mu \mathrm{g} \mathrm{L}{ }^{-1}$, respectively. Concentrations of $3.34 \mu \mathrm{g} \mathrm{kg}-1$ (dry basis) for trifloxystrobin and azoxystrobin (below the LOQ) were found in a sediment sample.
\end{abstract}

Keywords: pesticides; two-dimensional gas chromatography; micro-electron capture detection.

\section{INTRODUÇÃO}

A utilização de agrotóxicos nas produções agrícolas vem obtendo cada vez mais destaque no cenário mundial. O controle de pragas, através do uso de diferentes princípios ativos, reduziu o índice de doenças para os homens e animais e proporcionou aumento na produção agrícola. Contudo, resíduos desses compostos podem permanecer no ambiente por longos períodos e causar impactos nocivos a diferentes ecossistemas. Nesse contexto, o monitoramento desses compostos em diferentes matrizes como ar, água, solo, sedimento e alimentos se torna de extrema importância para que problemas de saúde pública sejam minimizados e/ou evitados. ${ }^{1}$

O estudo da ocorrência de agrotóxicos em sedimentos fornece informação a respeito da contaminação do corpo hídrico receptor. O sedimento se comporta como fonte de acumulação de poluentes (compostos orgânicos em geral e metais, por exemplo) e este comportamento se relaciona diretamente com sua constituição físico-química. Este fato confere ao sedimento grande importância no que tange à investigação de processos físicos, geoquímicos e biológicos no ambiente. ${ }^{2}$ As suas propriedades físico-químicas podem influenciar tanto no transporte e destino desses compostos no ambiente quanto no comportamento dos mesmos durante as etapas de extração, limpeza (clean up) e análise instrumental. A interação existente entre os agrotóxicos e o sedimento pode ser bastante forte, resultando em resíduos quimicamente ligados à matriz. Tendo em vista este contexto, esforços significativos vêm sendo realizados para o desenvolvimento de métodos eficientes que possibilitem a análise multirresíduo de agrotóxicos, minimizando etapas de extração e de clean up das amostras. ${ }^{3,4}$

Atualmente, técnicas rápidas e automatizadas para extração de amostras sólidas, que utilizam altas pressões e temperaturas, vêm sendo reportadas com destaque na literatura. Dentre estas, podem ser citadas a extração com fluido supercrítico (SFE, Supercritical Fluid Extraction), ${ }^{5-7}$ extração acelerada com solvente (ASE, Accelerated Solvent Extraction), ${ }^{8-10}$ extração assistida por micro-ondas (MAE,

*e-mail: elina@ufrgs.br
Microwave Assisted Extraction), ${ }^{11-13}$ ou técnica que utiliza forte agitação como a extração por ultrassom (USE, Ultrasonic Extraction) ${ }^{4,14,15}$ Outras técnicas como Soxhlet, ${ }^{16-18}$ extração em fase sólida (SPE, Solid-Phase Extraction) $)^{19,20}$ e microextração em fase sólida (SPME, Solid-Phase Micro Extraction) ${ }^{21-23}$ também são utilizadas para determinação de agrotóxicos em sedimentos. A extração por ultrassom é uma técnica bastante versátil que permite a extração de várias amostras simultaneamente, sem demandar o uso de materiais sofisticados. ${ }^{24} \mathrm{O}$ processo de extração por ultrassom proporciona um contato mais efetivo entre a matriz e o solvente, devido aos processos térmicos, mecânicos e de cavitação acústica. A exposição à irradiação ultrassônica faz com que haja formação de microbolhas, quando a pressão negativa exercida no sistema é alta o suficiente; sendo que, durante períodos de pressão positiva, as bolhas são comprimidas. Quando ocorre um colapso perto da superfície de um sólido, causado pela compressão e expansão das bolhas, as paredes do sólido podem ser danificadas e ter seu conteúdo (analitos) liberado para o meio. ${ }^{25}$

Atualmente, a cromatografia gasosa é a técnica mais utilizada para a determinação de agrotóxicos em diferentes matrizes. ${ }^{26-28} \mathrm{~A}$ cromatografia gasosa bidimensional abrangente $(\mathrm{GC} \times \mathrm{GC})$ é uma técnica relativamente recente, idealizada em 1991 por Liu e Phillips, ${ }^{29}$ e que apresenta grande poder de separação. $\mathrm{Na} \mathrm{GC} \times$ GC, duas colunas cromatográficas de diferentes mecanismos de retenção são acopladas em série, sendo a coluna da primeira dimensão $\left({ }^{1} \mathrm{D}\right)$ comumente de $30 \mathrm{~m}$ de comprimento e d.i. de 0,25 a 0,32 mm. A coluna da segunda dimensão ( $\left.{ }^{2} \mathrm{D}\right)$ deve ser mais curta, do tipo fast-GC, para que o perfil de separação obtido na ${ }^{1} \mathrm{D}$ seja mantido ${ }^{30} \mathrm{O}$ modulador é considerado o "coração" da técnica e é acoplado entre as duas colunas, sendo que sua função é de amostrar e focalizar as pequenas frações eluídas da primeira coluna e, em seguida, liberar essas porções, rapidamente, para a segunda coluna. ${ }^{31} \mathrm{O}$ período de amostragem corresponde ao período de modulação $\left(P_{M}\right)$, que é a duração de um ciclo completo de modulação, e ao tempo de separação na coluna da segunda dimensão. Devido ao tempo de separação da segunda dimensão ser muito curto, geralmente de 2 a $10 \mathrm{~s}$, a separação na segunda coluna é essencialmente isotérmica. ${ }^{32}$ Os picos gerados por um sistema $\mathrm{GC} \times \mathrm{GC}$ apresentam dois tempos de retenção, na primeira e segunda dimensão $\left({ }^{1} t_{R} e^{2} t_{R}\right.$, 
respectivamente). Essa característica proporciona grande vantagem analítica, pois se refere a dois mecanismos de separação, podendo ser utilizada com o propósito de identificação de compostos. ${ }^{33}$

O número de publicações científicas relacionado à determinação de agrotóxicos por $\mathrm{GC} \times \mathrm{GC}$ ainda é relativamente pequeno, mas suficiente para demonstrar as potenciais vantagens da técnica em relação à cromatografia monodimensional (1D-GC). As principais vantagens apresentadas pela GC $\times$ GC em relação à $1 \mathrm{D}-\mathrm{GC}$ são: aumento de resolução; aumento de sensibilidade devido à reconcentração da banda do soluto na ${ }^{2} \mathrm{D}$, favorecendo a detecção de componentes em nível de traços; estruturação do cromatograma 2D (bidimensional) de acordo com as características químicas dos compostos em análise e, aumento da capacidade de pico..$^{34,35}$

Trabalho recentemente publicado por este grupo, ${ }^{36}$ apresenta a otimização dos métodos cromatográficos de análise por GC- $\mu \mathrm{ECD}$ e GC $\times$ GC- $\mu$ ECD para determinação de agrotóxicos em sedimentos da região sob influência da cultura do arroz no estado do Rio Grande do Sul. Parâmetros experimentais como coluna cromatográfica, fluxos de gás de arraste e de make-up, temperatura do injetor, período de modulação, duração de pulso quente e diferença de temperatura entre fornos primário e secundário foram avaliados na busca das melhores condições analíticas. As coeluições observadas entre analitos e interferentes da matriz por $1 \mathrm{D}-\mathrm{GC}-\mu \mathrm{ECD}$ foram minimizadas pelo emprego da $\mathrm{GC} \times \mathrm{GC}-\mu \mathrm{ECD}$ devido ao seu maior potencial de separação, de forma a propiciar melhores resultados analíticos. A escolha do $\mu$ ECD foi devida a sua alta sensibilidade e seletividade para a determinação de compostos halogenados. Além disso, o uso deste detector também implica em menor custo de análise quando comparado ao uso de detector de espectrometria de massas por tempo de vôo. ${ }^{37}$ Estas características do $\mu \mathrm{ECD}$ aliadas ao potencial de separação da $\mathrm{GC} \times \mathrm{GC}$ propiciaram excelentes condições analíticas para a avaliação qualitativa de agrotóxicos em sedimentos. A Tabela 1 apresenta alguns trabalhos científicos que utilizaram a $\mathrm{GC} \times \mathrm{GC}$ como ferramenta analítica para determinação de agrotóxicos e outros poluentes organoclorados em diferentes matrizes.

Desta forma, este estudo teve por objetivo a validação de um método multirresíduo, por $\mathrm{GC} \times \mathrm{GC}-\mu \mathrm{ECD}$, para a determinação quantitativa de sete agrotóxicos (propanil, fipronil, propiconazol, trifloxistrobina, permetrina, difenoconazol e azoxistrobina) de diferentes classes químicas (anilida, pirazol, triazol, estrobilurina e piretroide) em sedimentos fluviais proveniente de regiões sob influência da cultura orizícola no estado do Rio Grande do Sul. O processo de validação foi realizado através da avaliação de parâmetros como curva de calibração e linearidade, precisão (repetitividade e precisão intermediária), exatidão (teste de recuperação) e limites de detecção e quantificação instrumentais.

Tabela 1. Aplicações da GC $\times$ GC a diferentes compostos organoclorados e agrotóxicos em várias matrizes

Analitos
8 agrotóxicos (organofosforado, anilida, pirazol, triazol, estrobilurina e piretroide)
Seis compostos da classe dos piretroides
Agrotóxicos organoclorados (OCPs), bifenilas policloradas (PCBs), éteres difenílicos policlorados
(PBDEs), dibenzo- -dioxinas policloradas (PCDDs), dibenzofuranos policlorados (PCDFs), naftalenos
policlorados (PCNs), terfenilas policloradas (PCTs) e toxafeno (CTT)
Cinco enantiômeros do toxafeno
Agrotóxicos organoclorados (OCPs), bifenilas policloradas (PCBs) e polibromadas (PBBs), éteres
difenílicos policlorados (PBDEs), dibenzo-p-dioxinas policloradas (PCDDs), dibenzofuranos poli-
clorados (PCDFs), naftalenos policlorados (PCNs), terfenils policlorados (PCTs), toxafeno (CTT),
naftalenos (PCNs) e dibenzotiofenos (PCDTs)

Nove fungicidas

Toxafeno

31 agrotóxicos organofosforados, piretroides e triazinas

$\begin{array}{ccc}\text { Tipo de amostra } & \text { Detecção } & \text { Ref. } \\ \text { Sedimento } & \mu E C D & 36 \\ \text { Uva } & \mu E C D & 38 \\ \text { Carne de porco, ovos e } & \mu E C D & 39 \\ \text { leite materno } & & \\ \text { Óleo de peixe } & \mu E C D & 40 \\ \text { Sedimento e poeira } & \mu E C D & 41\end{array}$

Éteres difenílicos policlorados, bifenilas policloradas (PCBs) e polibromadas (PBBs) e agrotóxicos organoclorados (OCPs)

20 agrotóxicos (organofosforados, organoclorados, carbamatos, dicarboximidas, benzimidazol, sulfito de alquila e piretroides)

36 agrotóxicos (organofosforados, organoclorados, carbamatos, dicarboximidas, piretroides)

\begin{tabular}{ccc} 
Vegetais & $\mu \mathrm{ECD}$ & 42 \\
Misturas técnicas & $\mu \mathrm{ECD}$ & 43 \\
Frutas & $\mu \mathrm{ECD}$ & 44 \\
Leite e soro humano & IDTOFMS & 45 \\
Maçã e pêssego & TOFMS & 46 \\
Chás de frutas, verde e & TOFMS & 47 \\
preto & & \\
Fumo & TOFMS & 48 \\
Cereal & TOFMS & 49 \\
Uva e vinho & TOFMS & 50 \\
Água de rio & TOFMS & 51 \\
Leite e nata & TOFMS & 52 \\
Mistura de padrões & FID & 53 \\
Soro humano & FID & 54 \\
\hline
\end{tabular}

FID: Detector de ionização por chama flame ionization detector. FPD: Detector fotométrico de chama, flame photometric detector. IDTOFMS: Espectrômetro de massas com analisador por tempo de vôo e diluição isotópica, isotope-dilution time-of-flight mass spectrometry 


\section{PARTE EXPERIMENTAL}

\section{Materiais e reagentes}

Os padrões dos agrotóxicos foram obtidos da Sigma-Aldrich (Seelze, Alemanha) com pureza superior a 97\%. Para as análises quantitativas foi utilizado o 3,4,5-tricloroguaiacol como padrão interno. As soluções padrão estoque foram preparadas pesando-se os agrotóxicos em frascos individuais em balança analítica de precisão (Shimadzu, AY220, Quioto, Japão) e dissolvendo-os em acetato de etila grau HPLC (Mallincrrodt, Phillipsburg, EUA) em balões volumétricos de $5 \mathrm{~mL}$, previamente silanizados. Todas as soluções foram guardadas em freezer à $-18^{\circ} \mathrm{C}$ em frascos de vidro âmbar silanizados. O sulfato de sódio anidro (Quimex, São Paulo, SP, Brasil), utilizado para retirar a umidade residual dos sedimentos, foi previamente seco em estufa a $400{ }^{\circ} \mathrm{C}$ por $4 \mathrm{~h}$ e armazenado em dessecador. Os papéis filtro utilizados foram de grau quantitativo (Quanty, Alemanha). O banho de ultrassom utilizado foi Maxiclean (Unique, Indaiatuba, SP, Brasil) com potência de $120 \mathrm{~W}$.

\section{Amostragem e preparação da amostra}

As coletas foram realizadas por técnicos da FEPAM (Fundação Estadual de Proteção Ambiental Henrique Luiz Roessler) na região da Bacia do Rio Santa Maria e na região do Rio Gravataí, no estado do Rio Grande do Sul. Foi coletado em média $1 \mathrm{~kg}$ de sedimento, com retirada de qualquer vegetação presente, próximo à margem, em locais de deposição de partículas finas (preferencialmente) e pouca profundidade. Os sedimentos foram coletados em frascos de vidro e transportados a $4{ }^{\circ} \mathrm{C}$, até o laboratório, onde foram congelados a $-18{ }^{\circ} \mathrm{C}$ em freezer, antes do processo de extração.

O processo de extração empregado foi baseado no método reportado por You e colaboradores. ${ }^{14} \mathrm{~A}$ água residual presente no sedimento foi retirada e o mesmo foi homogeneizado. Em seguida, foi parcialmente seco ao ar, à temperatura ambiente, em vidros de relógio. Para a etapa de extração, foram pesados $20 \mathrm{~g}$ de sedimento e adicionados $10 \mathrm{~g}$ de sulfato de sódio anidro, procedendo-se à homogeneização do material. Uma alíquota de $50 \mathrm{~mL}$ de uma solução $50: 50 \mathrm{v} / \mathrm{v}$ de acetona:diclorometano foi adicionada à amostra, a qual foi submetida à extração em 3 ciclos de 15 min no banho de ultrassom. $\mathrm{O}$ extrato foi levado à secura e o resíduo final dissolvido com $1,5 \mathrm{~mL}$ de acetato de etila.

Para os testes de recuperação foram adicionadas quantidades de 300,60 e $30 \mu \mathrm{L}$ de uma solução dos analitos a $10 \mathrm{mg} \mathrm{L}^{-1}$, para obtenção da fortificação a 150, 30 e $15 \mu \mathrm{g} \mathrm{kg}^{-1}$, respectivamente. As amostras foram deixadas em contato com a solução padrão contendo os agrotóxicos durante 24 h, após homogeneização e evaporação do solvente. Todos os solventes utilizados para extração foram de grau analítico (Vetec, Rio de Janeiro, RJ, Brasil) e bidestilados. A Tabela 2 apresenta as características e localização geográfica desses sedimentos.

\section{Condições cromatográficas}

As análises foram realizadas em um cromatógrafo Agilent 6890N (Agilent Technologies, Palo Alto, CA, EUA) com $\mu$ ECD, equipado com amostrador automático Combi PAL (CTC Analytics AG, Zwingen, Suíça). O cromatógrafo também possui um forno secundário (LECO, St. Joseph, MI, EUA) e um modulador térmico de dois estágios com quatro jatos (quad jet) de $\mathrm{N}_{2}$ (dois jatos quentes e dois frios, resfriados por $\mathrm{N}_{2}$ líquido). A coluna utilizada na primeira dimensão foi uma DB-5 (30 m x 0,25 mm x 0,25 $\mu \mathrm{m}$ ) e na segunda dimensão, DB-17ms (1,7 m x 0,18 mm x 0,18 $\mu \mathrm{m})$, ambas adquiridas da Agilent Technologies - J\&W Scientific (Palo Alto, CA, EUA). As temperaturas de injeção e detecção foram de 280 e $320^{\circ} \mathrm{C}$, respectivamente. $\mathrm{O}$ fluxo de gás de arraste $\left(\mathrm{H}_{2}, 99,999 \%\right.$, Linde Gás, Canoas-RS) foi de $2 \mathrm{~mL} \mathrm{~min}^{-1}$, enquanto que o fluxo de gás de make up $\left(\mathrm{N}_{2}, 99,999 \%\right.$, Linde Gás, Canoas-RS) foi de $150 \mathrm{~mL} \mathrm{~min}^{-1}$. O $P_{M}$ utilizado foi de $7 \mathrm{~s}$, com duração de pulso quente de $2,1 \mathrm{~s}$. A temperatura inicial do forno primário foi de $60{ }^{\circ} \mathrm{C}$ por $2,53 \mathrm{~min}$ e atingiu $180{ }^{\circ} \mathrm{C}$ a uma taxa de aquecimento de $35{ }^{\circ} \mathrm{C} \mathrm{min}^{-1}$, passando a $240{ }^{\circ} \mathrm{C}$ com taxa de aquecimento de $4{ }^{\circ} \mathrm{C} \mathrm{min}^{-1}$; tendo sido aumentada até $295{ }^{\circ} \mathrm{C}$, a $10{ }^{\circ} \mathrm{C} \mathrm{min}^{-1}$, onde permaneceu por $3 \mathrm{~min}$. A diferença de temperatura entre o forno primário e o secundário foi de $5{ }^{\circ} \mathrm{C}$. As injeções foram realizadas com volume de $1 \mu \mathrm{L}$, sem divisão de fluxo e com pulso de pressão de 60 psi por 29,4 s. As condições cromatográficas empregadas neste trabalho foram otimizadas em trabalho anterior. ${ }^{36}$

\section{Validação do método}

O processo de validação possibilita uma avaliação da eficiência de um processo analítico, quando do seu desenvolvimento, adaptação ou implementação. Este processo garante que um método analítico ofereça informações confiáveis e interpretáveis sobre uma amostra. ${ }^{56}$ Os parâmetros avaliados no processo de validação foram linearidade e curva de calibração, precisão (repetitividade e precisão intermediária), exatidão (testes de recuperação) e limites de detecção (LOD) e quantificação (LOQ). A curva de calibração foi avaliada através da injeção de soluções com concentrações na faixa de 5 a $415 \mu \mathrm{g} \mathrm{L}^{-1}$ (5 pontos escolhidos dentre as soluções de concentrações 5, 15, 35, $55,95,115,175,295,355$ e $\left.415 \mu \mathrm{g} \mathrm{L}^{-1}\right)$. Cada solução foi analisada cinco vezes. O composto 3,4,5-tricloroguaiacol foi utilizado como padrão interno na concentração de $100 \mu \mathrm{g} \mathrm{L}^{-1}$. A precisão do método foi verificada em termos de repetitividade e precisão intermediária, expressas como coeficiente de variação $(\mathrm{CV} \%)$. A repetitividade foi avaliada através da análise de 8 injeções de solução padrão a $100 \mu \mathrm{g} \mathrm{L}^{-1}$ em um único dia e mantendo-se constante as condições operacionais. A precisão intermediária foi verificada através da análise de 8 injeções da mesma solução padrão, mas em 2 dias diferentes. O CV\% foi calculado utilizando-se a média das áreas e alturas dos picos cromatográficos para cada agrotóxico. A exatidão de um processo analítico representa o quanto os resultados individuais, de um determinado ensaio, concordam com um valor de referência aceito

Tabela 2. Localização geográfica e características físico-químicas dos sedimentos analisados

\begin{tabular}{|c|c|c|c|c|c|c|}
\hline \multirow[t]{2}{*}{ Amostra } & \multirow[t]{2}{*}{ Localização geográfica } & \multicolumn{5}{|c|}{ Composição percentual } \\
\hline & & Carbono orgânico & Cascalho & Areia & Silte & Argila \\
\hline $\mathrm{SD}_{1}$ & $\begin{array}{l}31^{\circ} 8^{\prime} 11^{\prime \prime} \text { - latitude sul } \\
54^{\circ} 22^{\prime} 42^{\prime \prime}-\text { longitude oeste }\end{array}$ & 2,53 & 3,20 & 70,97 & 19,49 & 6,33 \\
\hline $\mathrm{SD}_{2}$ & $\begin{array}{l}30^{\circ} 16^{\prime} 49^{\prime \prime} \text { - latitude sul } \\
54^{\circ} 54^{\prime} 10^{\prime \prime} \text { - longitude oeste }\end{array}$ & 0,99 & 0 & 83,11 & 10,20 & 6,69 \\
\hline Amostra controle & $\begin{array}{l}29^{\circ} 56^{\prime} 10^{\prime \prime}-\text { latitude sul } \\
50^{\circ} 36^{\prime} 05^{\prime \prime}-\text { longitude oeste }\end{array}$ & 1,98 & 24,90 & 66,20 & 5,08 & 3,77 \\
\hline
\end{tabular}


como verdadeiro. Esse parâmetro mede o desvio do valor obtido em relação ao valor adotado como real ${ }^{56,57}$ e foi avaliado através dos testes de recuperação com amostra fortificada (amostra controle) nos níveis de 15,30 e $150 \mu \mathrm{g} \mathrm{kg}^{-1}$. O LOD pode ser definido como sendo a menor quantidade de um analito que pode ser detectada, mas não necessariamente quantificada, ou seja, é a menor concentração de um analito que pode ser diferenciada do ruído gerado por equipamentos que produzem linhas de base.$^{57} \mathrm{O}$ LOQ é a menor concentração do analito que pode ser determinada com um nível aceitável de exatidão e precisão. ${ }^{58}$

\section{RESULTADOS E DISCUSSÃO}

\section{Condições cromatográficas}

A Figura 1 apresenta o diagrama de cores em terceira dimensão (3D) obtido para os sete agrotóxicos com o jogo de colunas DB-5/ DB-17ms, onde é possível observar que os picos se encontram dispersos dentro do plano cromatográfico. Esse conjunto de colunas foi escolhido para o desenvolvimento do método analítico e sua aplicação, visto que apresentou melhor aproveitamento do plano cromatográfico, com ampla distribuição dos analitos na ${ }^{2} \mathrm{D}$, menor tempo de análise e boa simetria de pico, características importantes e desejáveis quando da análise de amostras complexas. ${ }^{36}$ Estes resultados confirmam o que é reportado na literatura, visto que a maioria dos trabalhos publicados apresenta a geometria convencional de colunas (fase estacionária

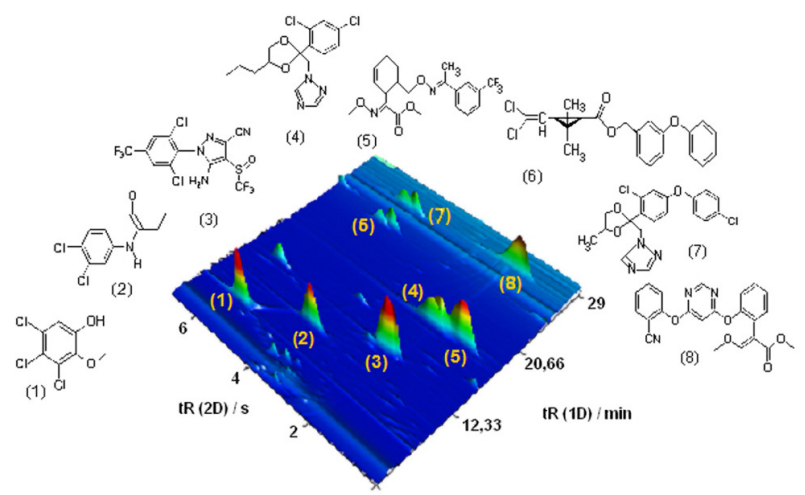

Figura 1. Diagrama de cores em $3 D$ obtido para a mistura de padrões a $100 \mu \mathrm{g} L^{-1}$ com estrutura química dos compostos analisados. (1) Tricloroguaiacol (PI), (2) Propanil, (3) Fipronil, (4) Propiconazol I e II, (5) Trifloxistrobina, (6) Permetrina cis e trans, (7) Difenoconazol I e II e (8) Azoxistrobina apolar na ${ }^{1} \mathrm{D}$ e polar na ${ }^{2} \mathrm{D}$ ) como sendo a que proporciona melhores resultados de análise para agrotóxicos em diversas matrizes. ${ }^{38,59}$

\section{Processo de extração por ultrassom}

O processo de extração por ultrassom foi baseado no método reportado por You e colaboradores. ${ }^{14}$ No trabalho descrito por esses autores foi utilizada uma etapa adicional de limpeza com $10 \mathrm{~g}$ de Florisil ativado e, posteriormente, parcialmente desativado com $6 \% \mathrm{~m} / \mathrm{v}$ de água destilada. Os autores obtiveram bons resultados de recuperação. Entretanto, testes realizados com Florisil durante este trabalho mostraram que para os sete agrotóxicos em estudo houve adsorção intensa dos analitos na coluna de Florisil, o que inviabilizou a utilização desta etapa de limpeza.

\section{Validação do método}

As equações da curva de calibração, coeficiente de determinação $\left(\mathrm{r}^{2}\right)$ e faixa linear de concentração são apresentados na Tabela 3.

Através das equações obtidas para as curvas analíticas pode-se observar que o método proposto apresentou ótima linearidade numa ampla faixa de concentração, com coeficientes de determinação $\left(r^{2}\right)$ entre 0,9920 e 0,9993 . Valores de $r^{2}$ acima de 0,98 são considerados satisfatórios ${ }^{60}$ Para a repetitividade, as áreas dos picos cromatográficos, para os sete compostos, apresentaram valores de coeficiente de variação de 0,64 a 3,78\%. Para as alturas dos picos, os valores situaram-se entre 0,77 e 3,16\%. Para a precisão intermediária, o coeficiente de variação relativo às áreas dos compostos situou-se entre 0,54 e $3,50 \%$ e para as alturas entre 0,81 e $3,74 \%$. Os tempos de retenção tiveram variação de $0,00 \%$ para a ${ }^{1} \mathrm{D}$ e de 0,11 a $0,66 \%$ para a ${ }^{2} \mathrm{D}$. Tendo em vista que os valores obtidos para repetitividade e precisão intermediária situaram-se abaixo de $20 \%,{ }^{56,61}$ o método cromatográfico proposto para a determinação dos sete compostos pode ser considerado preciso.

A Tabela 3 também apresenta os níveis de concentração encontrados para os testes de recuperação. O presente método mostrou-se eficiente (recuperação entre 50 e $120 \%$ e $\mathrm{CV} \leq 20 \%$ ) para todos os agrotóxicos no nível de concentração de $30 \mu \mathrm{g} \mathrm{kg}^{-1}$. Apesar da baixa recuperação obtida para a trifloxistrobina nos níveis de 15 e $150 \mu \mathrm{g} \mathrm{kg}^{-1}$ e para o propiconazol em $150 \mu \mathrm{g} \mathrm{kg}^{-1}$, o coeficiente de variação foi menor do que $20 \%$, o que indica que o método foi repetitivo. Para os compostos difenoconazol e azoxistrobina houve elevada recuperação (acima de $120 \%$ ) que pode ter sido causada por coeluições com interferentes da matriz, no nível de recuperação de $15 \mu \mathrm{g} \mathrm{kg}^{-1}$.

Tabela 3. Parâmetros de mérito, níveis de recuperação em base seca e coeficientes de variação para os compostos estudados em sedimento por GC $\times$ GC- $\mu$ ECD

\begin{tabular}{|c|c|c|c|c|c|c|c|c|c|c|c|}
\hline \multirow{3}{*}{ Analito } & \multicolumn{6}{|c|}{ Recuperação por nível de fortificação } & \multirow{2}{*}{\multicolumn{5}{|c|}{ Parâmetros de mérito }} \\
\hline & \multicolumn{2}{|c|}{$15 \mu \mathrm{g} \mathrm{kg}^{-1}$} & \multicolumn{2}{|c|}{$30 \mu \mathrm{g} \mathrm{kg}^{-1}$} & \multicolumn{2}{|c|}{$150 \mu \mathrm{g} \mathrm{kg}^{-1}$} & & & & & \\
\hline & Rec & $\mathrm{CV}$ & $\operatorname{Rec}$ & $\mathrm{CV}$ & $\operatorname{Rec}$ & $\mathrm{CV}$ & Equação da Reta & $\mathrm{r}^{2}$ & Linearidade $\left(\mu \mathrm{g} \mathrm{L}^{-1}\right)$ & LOD & LOQ \\
\hline Propanil & 74 & 13 & 67 & 9 & 60 & 20 & $y=0,0023 x+0,0186$ & 0,9992 & $5-295$ & 1,07 & 3,23 \\
\hline Fipronil & 64 & 14 & 67 & 11 & 65 & 18 & $y=0,0061 x+0,0756$ & 0,9945 & $5-415$ & 0,26 & 0,77 \\
\hline Propiconazol a & 54 & 7 & 61 & 9 & 47 & 18 & $y=0,0044 x-0,0234$ & 0,9920 & $15-295$ & 0,08 & 0,25 \\
\hline Trifloxistrobina & 40 & 9 & 52 & 9 & 42 & 16 & $y=0,0029 x+0,0061$ & 0,9993 & $5-415$ & 0,25 & 0,74 \\
\hline Permetrina $^{\mathrm{b}}$ & 133 & 20 & 52 & 30 & 39 & 23 & $y=0,001 x+0,0013$ & 0,9990 & $5-295$ & 0,19 & 0,58 \\
\hline Difenoconazol & 254 & 15 & 115 & 9 & 92 & 24 & $y=0,0014 x-0,0058$ & 0,9942 & $5-415$ & 0,64 & 1,94 \\
\hline Azoxistrobina & 127 & 14 & 78 & 9 & 62 & 20 & $y=0,0027 x+0,0092$ & 0,9963 & $5-415$ & 0,35 & 1,06 \\
\hline
\end{tabular}

${ }^{\mathrm{a}}$ Soma dos estereoisômeros; ${ }^{\mathrm{b}}$ Soma dos isômeros cis e trans; $\mathrm{Rec}=$ recuperação percentual; $\mathrm{CV}=$ coeficiente de variação percentual; LOD = limite de detecção $\left(\mu \mathrm{g} \mathrm{L}{ }^{-1}\right)$; LOQ = limite de quantificação $\left(\mu \mathrm{g} \mathrm{L}^{-1}\right)$. 
Para o difenoconazol no nível de fortificação de $150 \mu \mathrm{g} \mathrm{kg}^{-1}$ e para a permetrina, no nível de $30 \mu \mathrm{g} \mathrm{kg}^{-1}$, houve boa recuperação, mas a precisão do método não foi satisfatória $(>20 \%)$. A resposta relativa obtida para alguns compostos no ECD, como os hidrocarbonetos, é de 0,01 (tendo como base o clorobenzeno com resposta igual a 1). ${ }^{62}$ Caso ocorra coeluição dos agrotóxicos estudados com esses compostos, por exemplo, pode ocorrer uma contribuição negativa na resposta analítica, com diminuição de intensidade de sinal. No banho de ultrassom o transdutor é diretamente preso no fundo da cuba do aparelho e a energia ultrassonora é transmitida através da água. ${ }^{63}$ Com isso, há muita dispersão de energia e, consequentemente, maior probabilidade de geração de pontos de maior e menor intensidade de extração. O sinergismo, causado pelo efeito negativo no sinal (sinal diminuído por coeluições com compostos de baixa resposta relativa no ECD) e pela alta variabilidade no processo de extração no ultrassom, pode ter contribuído para que alguns resultados de recuperação não fossem tão satisfatórios. O emprego de uma sonda de ultrassom poderia melhorar a repetibilidade do processo de extração, como já constatado por You e colaboradores. ${ }^{14}$ A Figura 2 apresenta o diagrama tridimensional e os cromatogramas reconstruídos da ${ }^{1} \mathrm{D}$ $\mathrm{e}^{2} \mathrm{D}$ para amostra de sedimento fortificada.

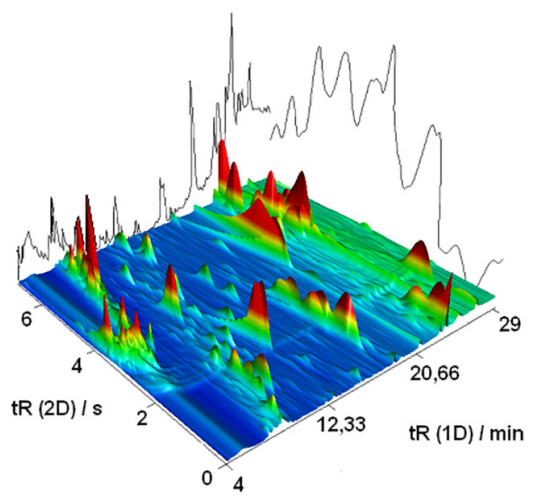

Figura 2. Diagrama de cores em $3 D$ da amostra fortificada a $15 \mu \mathrm{g} \mathrm{kg}^{-1} \mathrm{com}$ reconstrução dos cromatogramas da ${ }^{1} \mathrm{D} e^{2} \mathrm{D}$

Através da Figura 2 é possível observar o perfil cromatográfico da amostra, onde se destaca a grande quantidade de compostos detectados, apesar da utilização de um detector seletivo. A complexidade da amostra de sedimento evidencia que a utilização da técnica monodimensional (GC- $\mu \mathrm{ECD}$ ) não seria suficiente para obter separação entre os analitos e os constituintes da matriz. A Tabela 3 também contém os limites instrumentais de detecção e quantificação para os agrotóxicos em estudo. Os limites de detecção e quantificação instrumentais variaram de 0,08 a 1,07 e de 0,25 a $3,23 \mu \mathrm{g} \mathrm{L}^{-1}$, respectivamente. Para fins de ilustração, já que não existe legislação vigente que contemple esses agrotóxicos em sedimentos, os LMR dos analitos para a matriz arroz variam de 0,01 a $2 \mathrm{mg} \mathrm{kg}^{-1},{ }^{64}$ evidenciando que os limites encontrados foram considerados satisfatórios. Existem poucos trabalhos na literatura que trazem resultados quantitativos para determinação de agrotóxicos por GC $\times$ GC. Pizzutti e colaboradores $^{38}$ desenvolveram um modulador a ar comprimido e avaliaram o seu potencial através da determinação de 6 agrotóxicos da classe dos piretroides em uva e padrões de $n$-alcanos. Os LOD e LOQ instrumentais para os agrotóxicos ficaram na faixa de 0,003 a 0,006 e de 0,01 a $0,02 \mathrm{mg} \mathrm{L}^{-1}$, respectivamente. Ramos e colaboradores $^{44}$ avaliaram o potencial da GC $\times$ GC- $\mu$ ECD aliada à técnica de dispersão da matriz em fase sólida (MSPD) para determinação de 31 agrotóxicos (organofosforados, triazinas e piretroides) em frutas. Os autores também utilizaram a cromatografia monodimensional (GC/qMS) e compararam os resultados obtidos. O limite de detecção para a $\mathrm{GC} \times \mathrm{GC}$ ficou na faixa de 0,005 a $3,6 \mu \mathrm{g} \mathrm{kg}^{-1}$, enquanto que para GC/qMS os valores se situaram na faixa de 9 a $250 \mu \mathrm{g} \mathrm{kg}^{-1}$, o que evidencia uma maior sensibilidade da técnica $\mathrm{GC} \times \mathrm{GC}-\mu \mathrm{ECD}$.

\section{Aplicabilidade do método a amostras reais}

A aplicabilidade do método de extração foi avaliada através da análise de duas amostras de sedimentos $-\mathrm{SD}_{1}$ e $\mathrm{SD}_{2}$. Foram encontrados resíduos dos agrotóxicos trifloxistrobina e azoxistrobina somente na amostra $\mathrm{SD}_{2}$. O valor de concentração encontrado para trifloxistrobina foi de $3,34 \mu \mathrm{g} \mathrm{kg}^{-1}$, em base seca e para azoxistrobina a concentração ficou abaixo do LOQ. A confirmação da presença de um determinado analito em uma amostra pode ser realizada através do uso do detector de espectrometria de massas ou de injeções em colunas cromatográficas cujas fases estacionárias propiciem a separação através de distintos mecanismos de separação, caso não exista a disponibilidade de um detector de espectrometria de massas. ${ }^{14}$ Sendo assim, a confirmação da presença dos resíduos de azoxistrobina e trifloxistrobina foi realizada através de coinjeções em colunas de fases estacionárias diferentes, com o jogo de colunas HP-50+/DB$1 \mathrm{~ms}$, utilizando-se o $\mu \mathrm{ECD}$.

\section{CONCLUSÃO}

O método de análise de agrotóxicos proposto apresentou ótimos resultados, evidenciando o potencial da técnica de $\mathrm{GC} \times \mathrm{GC}$ para a análise de poluentes em nível de traços em matrizes complexas. A alta capacidade de pico da técnica contribuiu para que o processo de quantificação tivesse pouca influência de coeluições de interferentes da matriz com os analitos em estudo, apesar de as amostras não terem sido submetidas a uma etapa de limpeza. Além disso, os valores encontrados para os limites de detecção e quantificação comprovam a grande sensibilidade da técnica.

Este trabalho amplia o uso da GC $\times$ GC para mais uma situação específica de relevante importância ambiental, visto que o número de aplicações desta técnica ainda é pequeno na literatura científica. O método em questão pode ser empregado por laboratórios de instituições governamentais de fiscalização ou por outros laboratórios prestadores de serviço, contribuindo assim para obtenção de informações relevantes sobre estes compostos no meio ambiente ou sobre um possível mau gerenciamento de sua aplicação nas lavouras. Informações obtidas por métodos analíticos como este podem minimizar riscos de poluição do meio ambiente e, também, riscos à saúde da população.

\section{AGRADECIMENTOS}

Ao Conselho Nacional de Desenvolvimento Científico e Tecnológico (CNPq), à Fundação Estadual de Proteção Ambiental (FEPAM) e Coordenação de Aperfeiçoamento de Pessoal de Nível Superior (CAPES) pelo suporte financeiro a este projeto.

\section{REFERÊNCIAS}

1. Flores, A. V.; Ribeiro, J. N.; Neves, A. A.; Queiroz, E. L. R.; Amb. Soc. 2004, 7, 111.

2. Secco, T.; Pellizzato, F.; Sfriso, A.; Pavoni, B.; Chemosphere 2005, 58, 279.

3. Smalling, K. L.; Kuivila, K. M.; J. Chromatogr., A 2008, $1210,8$.

4. Vagi, M. C.; Petsas, A. S.; Kostopoulou, M. N.; Karamanoli, M. K.; Lekkas, T. D.; Desalination 2007, 210, 146.

5. Schantz, M. M.; Bowadt, S.; Jr Benner, B. A.; Wise, S. A.; Howthorne, S. B.; J. Chromatogr., A 1998, 816, 213. 
6. Robertson, A. M.; Lester, J. N.; Environ. Sci. Technol. 1994, 28, 346.

7. Carrasco, P. B.; Díez, S.; Jiménez, J.; Marco, M. P.; Bayona, J. M.; Water Res. 2003, 37, 3658

8. Dabrowski, L.; Mozajska, H. G.; Biziuk, M.; Gaca, J.; Namiesnik, J.; J. Chromatogr., A 2002, 957, 59.

9. Graña, E. C.; Carou, M. I. T.; Lorenzo, S. M.; Mahía, P. L.; Rodríguez, D. P.; Fernández, E. F.; Chemosphere 2006, 64, 588.

10. Villaverde, J.; Hildebrandt, A.; Martínez, E.; Lacorte, S.; Morillo, E.; Maqueda, C.; Viana, P.; Barceló, D.; Sci. Total Environ. 2008, 390, 507.

11. Numata, M.; Yarita, T.; Aoyagi, Y.; Takatsu, A.; Anal. Sci. 2004, $20,793$.

12. Camel, V.; Trends Anal. Chem. 2000, 19, 229.

13. Gfrerer, M.; Lankmayer, E.; Anal. Chem. Acta 2005, 533, 203.

14. You, J.; Weston, D. P.; Lydy, M. J.; Arch. Environ. Contam. Toxicol. 2004, 47, 141.

15. Xue, N.; Zhang, D.; Xu, X.; Water Res. 2006, 40, 183.

16. Woudneh, M. B.; Oros, D. R.; J. Chromatogr., A 2006, 1135, 71.

17. Darko, G.; Akoto, O.; Oppong, C.; Chemosphere 2008, 72, 21.

18. Covaci, A.; Gheorghe, A.; Hulea, O.; Schepens, P.; Environ. Pollut. 2006, 140, 136.

19. Peñuela, G.A.; Barceló, D.; J. Chromatogr., A 1998, 795, 93.

20. D browska, H.; D browski, Ł.; Biziuk, M.; Gaca, J.; Namie nik, J.; J. Chromatogr., A 2003, 1003, 29.

21. Aguilar, C.; Peñalver, S.; Pocurull, E.; Borrull, F.; Marcé, R. M.; J. Chromatogr., A 1998, 795, 105.

22. Diaz, A.; Vásquez, L.; Ventura, F.; Galceran, M. T.; Anal. Chim. Acta 2004, 506, 71.

23. Chang, S.; Doong, R.; Chemosphere 2006, 62, 1869.

24. Gonçalves, C.; Alpendurada, M. F.; Talanta 2005, 65, 1179.

25. Pan, J.; Xia, X. X.; Liang, J.; Ultrason. Sonochem. 2008, 15, 25.

26. van der Hoff, R. G.; van Zoonen, P.; J. Chromatogr., A 1999, 843, 301.

27. Tankiewicz, M.; Fenik, J.; Biziuk, M.; Trends Anal. Chem. 2010, 29, 1050.

28. Feo, M. L.; Eljarrat, E.; Barceló, D.; Trends Anal. Chem. 2010, 29, 692.

29. Liu, Z. Y.; Phillips, J.; J. Chromatogr. Sci. 1991, 29, 227.

30. Pedroso, M. P.; Godoy, L. A. F.; Fidélis, C. H. V.; Ferreira, E. C.; Poppi, R. J.; Augusto, F.; Quim. Nova 2009, 32, 421.

31. Górecki, T.; Harynuk, J.; Pani , O.; J. Sep. Sci. 2004, 27, 359.

32. Pegasus HT and ChromaTOF Software Instruction Manual, Version 3.3, Leco, St. Joseph, MI, 2007.

33. Phillips, J. B.; Xu, J.; J. Chromatogr., A 1995, 703, 327.

34. Phillips, J. B.; Beens, J.; J. Chromatogr., A 1999, 856, 331.

35. Mondello, L.; Tranchida, P. Q.; Dugo, P.; Dugo, G.; Mass Spectrom. Rev. 2008, 27, 101

36. Silva, J. M.; Zini, C. A.; Canizares, E. M. P. N.; Leal, K, A.; Caramão, E. B.; Quim. Nova 2010, 33, 591.

37. Koritár, P.; Haglund, P.; de Boer, J.; Brinkiman, U. A. Th.; Trends Anal. Chem. 2006, 25, 373.

38. Pizzutti, I. R.; Vreuls, R. J. J.; Kok, A.; Roehrs, R.; Martel, S.; Friggi, C. A.; Zanella, R.; J. Chromatogr., A 2009, 1216, 3305.

39. Bordajandi, L. R.; Ramos, J. J.; Sanz, J.; González, M. J.; Ramos, L.; J. Chromatogr., A 2008, 1186, 312.
40. Bordajandi, L. R.; Ramos, L.; González, M. J.; J. Chromatogr., A 2006, $1125,220$.

41. Korytár, P.; Leonards, P. E. G.; de Boer, J.; Brinkiman, U. A. Th.; J. Chromatogr., A 2005, 1086, 29.

42. Khummueng, W.; Trenerry, C.; Rose, G.; Marriott, P. J.; J. Chromatogr., A 2006, 1131, 203.

43. Korytár, P.; van Stee, L. L. P.; Leonards, P. E. G.; de Boer, J.; Brinkman, U. A. Th.; J. Chromatogr., A 2003, 994, 179.

44. Ramos, J. J.; González, M. J.; Ramos, L.; J. Chromatogr., A 2009, 1216, 7307.

45. Focant, J. F.; Sjdin, A.; Turner, W. E.; Patterson, D. G.; Anal. Chem. 2004, 76, 6313.

46. Zrostlíková, J.; Hajslová, J.; Cajka, T.; J. Chromatogr., A 2003, 1019, 173.

47. Schurek, J.; Portolés, T.; Hajslova, J.; Riddellova, K.; Hernández, F.; Anal. Chem. Acta 2008, 611, 163.

48. Cochran, J.; J. Chromatogr., A 2008, 1186, 202.

49. van der Lee, M. K.; van der Weg, G.; Traag, W. A.; Mol, H. G. J.; J. Chromatogr., A 2008, 1186, 325.

50. Dasgupta, S.; Banerjee, K.; Patil, S. H.; Ghaste, M.; Dhumal, K. N.; Adsule, P. G.; J. Chromatogr., A 2010, 1217, 3881.

51. Matamoros, V.; Jover, E.; Bayona, J. M.; Anal. Chem. 2010, 82, 699.

52. Hayward, D. G.; Pisano, T. S.; Wong, J. W.; Scudder, R. J.; J. Agric. Food Chem. 2010, 58, 5248.

53. Ong, R.; Marriott, P.; Morrison, P.; Haglund, P.; J. Chromatogr., A 2002, 962, 135.

54. Liu, Z.; Sirimanne, S. R.; Patterson, D. G.; Needham, L.; Phillips, J. B.; Anal. Chem. 1994, 66, 3086.

55. Chin, S. T.; Wu, Z. Y.; Morrison, P. D.; Marriott, P. J.; Anal. Methods 2010, 2, 243.

56. Ribani, M.; Bottoli, C. B. G.; Collins, C. H.; Jardim, I. C. S. F.; Melo, L. F. C.; Quim. Nova 2004, 27, 771.

57. Lanças, F. M.; Validação de Métodos Cromatográficos, $1^{\mathrm{a}}$ ed., Rima: São Carlos, 2006.

58. INMETRO; Orientação Sobre Validação de Métodos de Ensaios Químicos, 2007.

59. Banerjee, K.; Patil, S. H.; Dasgupta, S.; Oulkar, D. P.; Patil, S. B.; Savant, R.; Adsule, P. G.; J. Chromatogr., A 2008, $1190,350$.

60. Chasin, A. A. M.; Nascimento, E. S.; Ribeiro Neto, L. M.; Siqueira, M. E. P. B.; Andraus, M. H.; Salvatori, M. C.; Fernícola, N. A. G.; Gorni, R.; Salcedo, S.; Rev. Bras. Toxicol. 1998, 11, 1.

61. Brito, N. M.; Junior, O. P. A.; Polese, L.; Ribeiro, M. L.; Pesticidas: Rev. Ecotox. Meio Ambiente 2003, 13, 129.

62. Grob, R. L.; Barry, E. F.; Modern Practice of Gas Chromatography, $4^{\text {th }}$ ed., Wiley: Hoboken, 2004.

63. Barboza, J. C. S.; Serra, A. A.; Quim. Nova 1992, 15, 301.

64. http://www.anvisa.gov.br/toxicologia/monografias/index.htm, acessada em Novembro 2007. 